\title{
Minor congenital variations of cusp size in tricuspid aortic valves Possible link with isolated aortic stenosis
}

\author{
F. E. M. G. VOLLEBERGH AND A. E. BECKER \\ From the Department of Pathology, University of Amsterdam, Wilhelmina Gasthuis, Amsterdam, The \\ Netherlands
}

Clinically isolated aortic stenosis is most commonly caused by a congenitally malformed aortic valve. Many elderly patients with isolated aortic stenosis have a tricuspid aortic valve. It has been suggested in the past that inequalities in individual cusp size could be a factor leading 10 the development of the stenoses in these cases.

In this study the width and height of individual aortic valve cusps were measured at necropsy in 200 hearts of patients without clinical signs of aortic stenosis, though the majority of valves obtained from elderly persons showed some ageing changes. The results were compared with similar measurements in 16 hearts of patients with clinically diagnosed and necropsy proven isolated aortic stenosis.

The results show that inequality in cusp size is the rule rather than the exception. Indeed, only 5 of the 200 'normal' tricuspid aortic valves had 3 cusps of identical height and width, and each of the 16 cases with isolated aortic stenosis had cusps of differing size.

The results seem to contradict the aforementioned hypothesis. However, the striking similarities between isolated aortic stenosis and the ageing aortic valve, suggest that isolated tricuspid aortic valve stenosis is an extreme within a spectrum of ageing alterations. Inequality in cusp size, with differences in mechanical tissue stress as a consequence, could then play a role in the pathogenesis of the stenosis by accelerating the ageing process.

Clinically isolated aortic stenosis is most commonly caused by a congenitally malformed valve, either unicuspid or bicuspid in nature (Roberts, 1970a). The former is considered intrinsically stenotic, whereas the latter may show early degenerative changes probably initiated by haemodynamic forces, which lead to fibrosis and calcification (Edwards, 1961; Roberts, 1970b).

Nevertheless, there remains a good percentage of patients with isolated valvar aortic stenosis in whom the valve is tricuspid. The pathogenesis of this type of aortic stenosis is as yet unsolved. Most investigators agree that the microscopically observed changes within the valve are compatible with those seen in ageing, but it is not known why the valve should become so severely affected that stenosis results (Mönckeberg, 1904; Sohval and Gross, 1936; Hultgren, 1948; McMillan and Lev, 1964; Sell and Scully, 1965; Kim and Huang, 1971; Roberts et al., 1971; Kim et al., 1976). The similarities with the acquired condition in conReceived for publication 28 January 1977 genitally bicuspid aortic valve have led Roberts (1970a) to pose the question, 'could the aortic valve be tricuspid and still congenitally malformed?' He suggested that inequality in cusp size could account for early cusp degeneration and ultimate stenosis. Indeed, at least 3 of 49 patients in his series of isolated aortic stenosis clearly had aortic valve cusps of unequal size.

In an attempt to answer this question, we have studied the variation in sizes of aortic valve cusps in the normal heart and in 16 patients with isolated aortic stenosis.

\section{Subjects and methods}

Two hundred normal hearts were selected from routine necropsies on patients who had no clinical signs of aortic stenosis and no other cardiac abnormality that could have affected the aortic valve. Each of the hearts presented a clearly delineated tricuspid aortic valve. Specimens from elderly patients showed ageing changes of the aortic valve, 


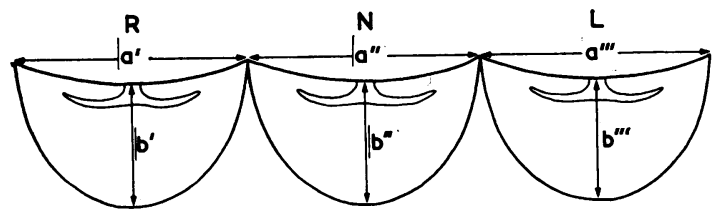

Fig. 1 Schematic drawing of the three semilunar cusps of the aortic valve, illustrating the way in which measurements for width and height have been taken. The maximal width (a) is represented by the distance between the two commissural insertions of a single cusp, measured along a line covering the inner wall of the aorta. The maximal height (b) is represented by the distance between the base of the cusp and its free rim, measured along a line perpendicular to line a and through the central part of the cusp, covering its ventricular surface. These measurements have been taken for each of the three cusps $\left(a^{\prime}-^{\prime \prime \prime}, b^{\prime}-^{\prime \prime \prime}\right)$.

characterised by cusp thickening, minor calcific deposits, and varying degrees of fusion of commissures. However, in each instance accurate measurements of the valve cusps could still be obtained. The youngest patient was 7 years old and the oldest patient was 93 years of age; the average age was 62.4 years. Fifty-four patients were less than 40 years of age. One-hundred and fifteen of the patients were male and 85 were female.

The 16 hearts with isolated aortic valve stenosis were derived from 9 male and 7 female patients, who had shown clinical signs of aortic stenosis: and confirmed by intracardiac catheterisation studies. The ages ranged from 58 to 86 years, with an average of 72.5 years. In each instance necropsy showed that only the aortic valve was malformed. None of these cases showed signs of rheumatic disease. The 16 hearts with isolated aortic stenosis were selected on the basis that each presented only minor degrees of calcification, so that accurate measurment of the individual valve cusps was possible.

In each heart the aortic valve was measured in an unfixed state. For each cusp the width and maximal height were measured (Fig. 1).

\section{Results}

\section{NORMAL HEARTS}

The average width for the right, non-, and left coronary cusps, among 200 hearts, was calculated as $25.9,25.5$, and $25.0 \mathrm{~mm}$, respectively. For each cusp the absolute width was expressed as a percentage of the width of a neighbouring cusp (Fig. 2). The right coronary cusp, compared with the left coronary cusp, varied between 76 and 159 per cent, the non-coronary cusp compared with the right coronary cusp varied between 62 and 162 per cent, and the figures for the left coronary cusp compared with the non-coronary cusp varied between 62 and 150 per cent.

For the height of the cusps similar variations were found. The average height for the right, non-, and left coronary cusps was calculated as $14 \cdot 1,14 \cdot 1$, and $14.2 \mathrm{~mm}$, respectively. When the average height was expressed as a percentage of the average width for each cusp, the percentages were 54, 55,

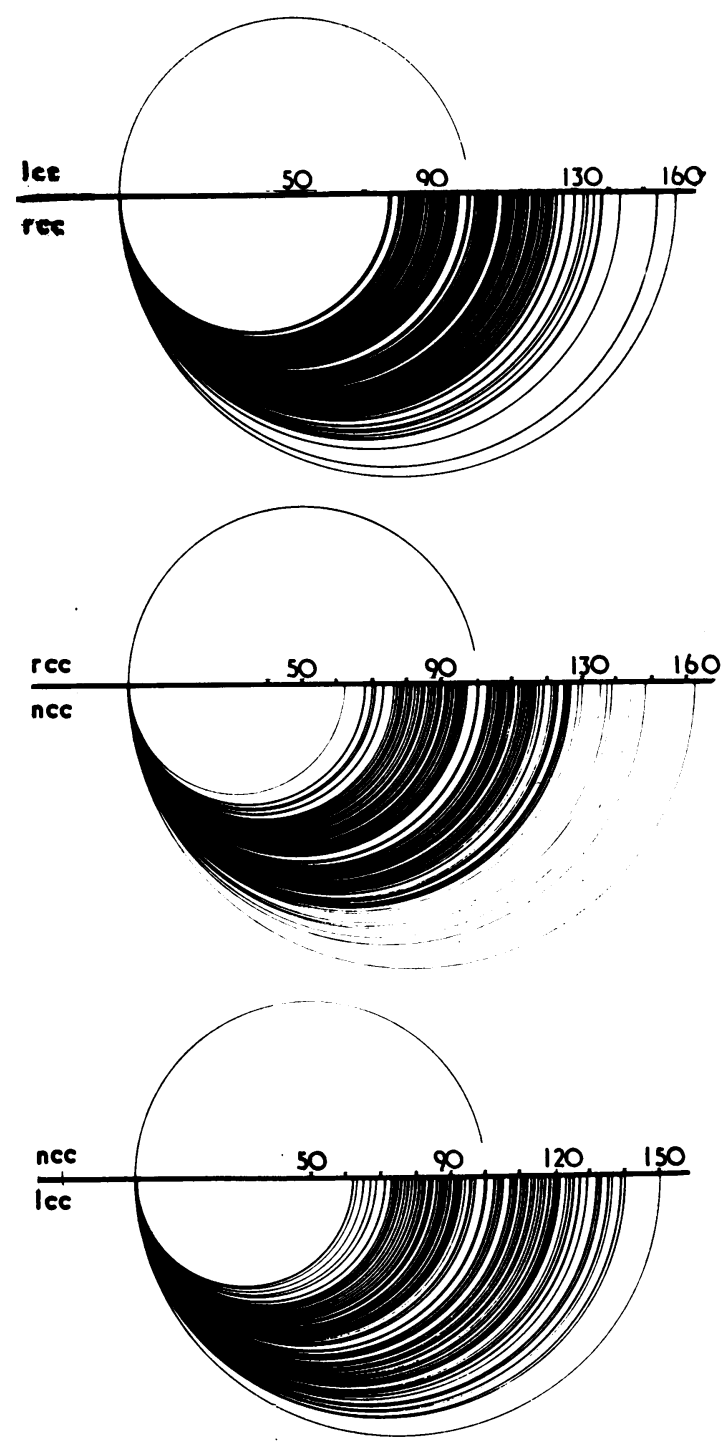

Fig. 2 Two hundred normal hearts. The absolute width of each cusp has been expressed as a percentage of a neighbouring cusp (rcc, right coronary cusp; ncc, non-coronary cusp; lcc, left coronary cusp). 


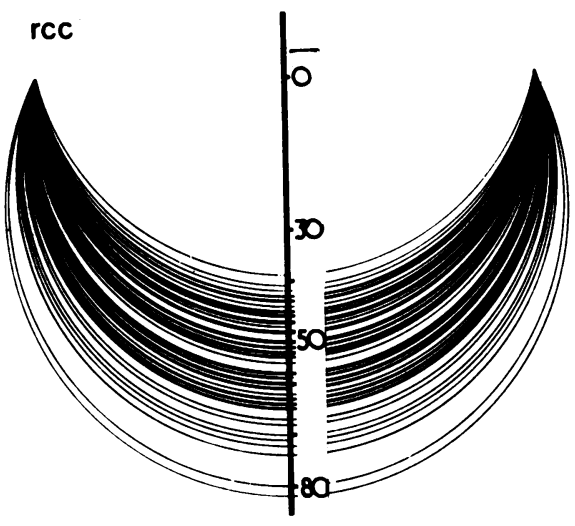

ncc

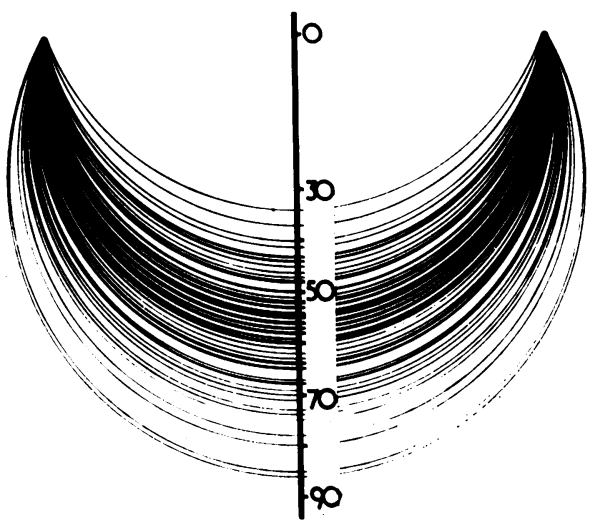

ICC

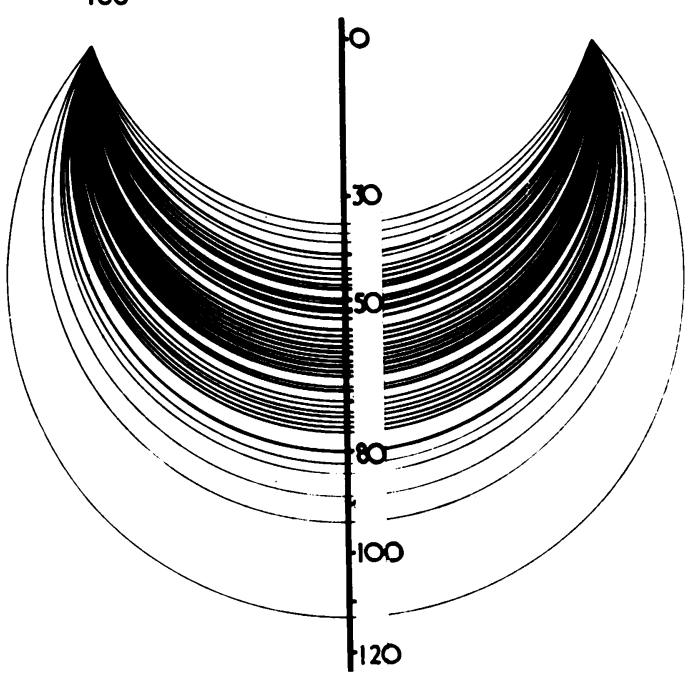

Fig. 3 Two hundred normal hearts. The absolute height of each cusp has been expressed as a percentage of the width of the same cusp. and 57 per cent, respectively. The absolute height of the individual cusps, again expressed as a percentage of the width of the same cusp, showed distinct differences (Fig. 3). For the right coronary cusp the height varied between 39 and 82 per cent, the height of the non-coronary cusp varied between 34 and 87 per cent, and the left coronary cusp varied between 34 and 113 per cent.

The surface area of each cusp was approximated to the nearest square millimetre by applying the formula $\frac{\pi}{4} a b$, derived from the ellipse, using the absolute figures representing width (a) and height (b). The cusp with the largest proportional area was thus identified for each heart and the hearts were classified accordingly. This indicated that only 5 hearts among the 200 specimens had 3 cusps of equal size (Fig. 4). Inequality in cusp sizes was present in 53 of the 54 hearts of patients less than 40 years of age, i.e. hearts without any grossly appreciable ageing changes.

\section{ISOLATED AORTIC VALVE STENOSIS}

The average width of the right, non-, and left coronary cusps, calculated on the basis of the 16 hearts studied was $26.8,26.0$, and $24.4 \mathrm{~mm}$, respectively. When the absolute width of each cusp was expressed as a percentage of a neighbouring
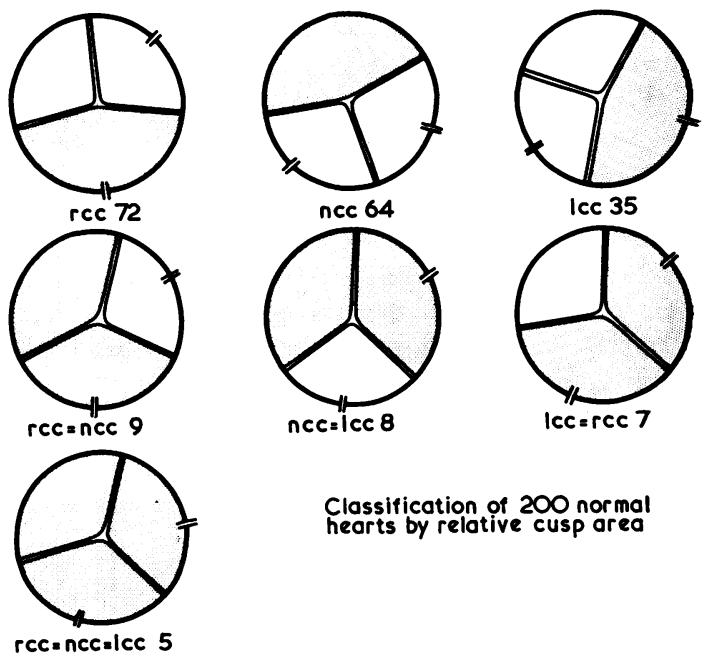

Classification of 200 normal hearts by relative cusp area

Fig. 4 Two hundred normal hearts. The surface area of each cusp was calculated (see text) from the absolute figures, representing width and height. The cusp(s) with the largest proportional area (shaded) was thus identified for each heart and they have been classified accordingly. Only five hearts had three equally sized cusps. 

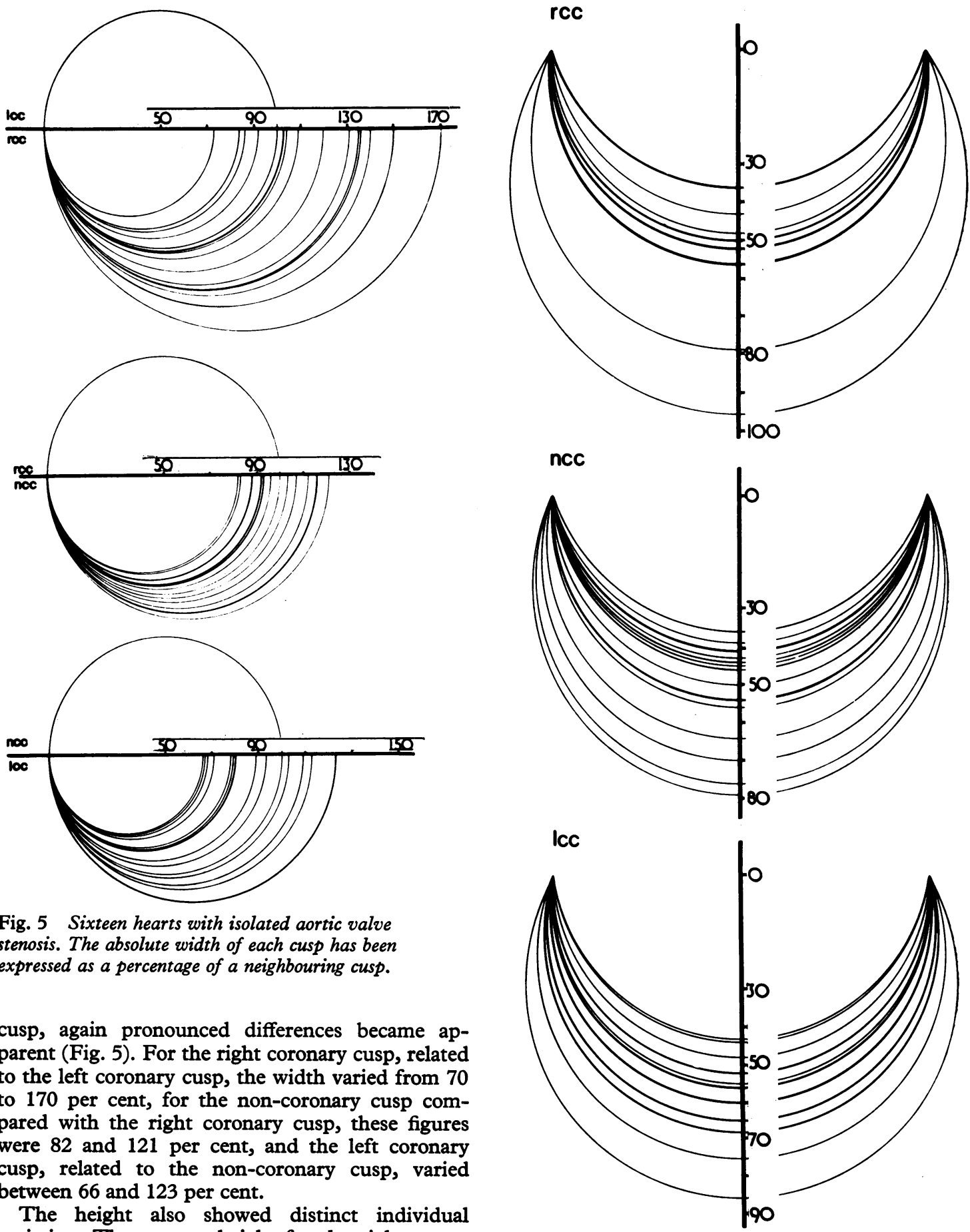

Fig. 5 Sixteen hearts with isolated aortic valve stenosis. The absolute width of each cusp has been expressed as a percentage of a neighbouring cusp.

cusp, again pronounced differences became apparent (Fig. 5). For the right coronary cusp, related to the left coronary cusp, the width varied from $\mathbf{7 0}$ to 170 per cent, for the non-coronary cusp compared with the right coronary cusp, these figures were 82 and 121 per cent, and the left coronary cusp, related to the non-coronary cusp, varied between 66 and 123 per cent.

The height also showed distinct individual variation. The average height for the right, non-, and left coronary cusps was calculated as 13.9, 13.6 , and $14.0 \mathrm{~mm}$, respectively. When expressed as a percentage of the average width the figures

Fig. 6 Sixteen hearts with isolated aortic valve stenosis. The absolute height of each cusp has been expressed as a percentage of the width of the same cusp. 


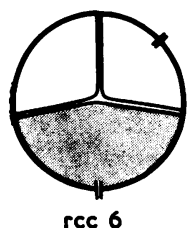

nec 5

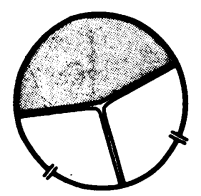

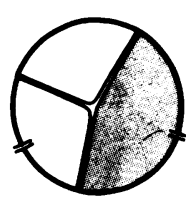

$\operatorname{lcc} 5$
Classification of 16 hearts with senile calcific oortic stenosis

Fig. 7 Sixteen hearts with isolated aortic stenosis. Classification of the area of the cusps (see legend to Fig. 4) indicates that all 16 hearts possessed cusps of unequal sizes.

were 52,52 , and 57 per cent, respectively. For each individual cusp the height, again expressed as a percentage of the width of the cusp, showed obvious variations (Fig. 6). For the right coronary cusp the height varied between 36 and 96 per cent, for the non-coronary cusp the figures varied from 35 to 79 per cent, and for the left coronary cusp the figures varied between 43 and 86 per cent.

None of the 16 hearts with isolated aortic stenosis had cusps of equal size (Fig. 7).

In each instance fusion of commissures had occurred, though not to the same extent at all sites. A pronounced fusion was considered to be present when two valve cusps had commissural valve adherence over a distance of at least $5 \mathrm{~mm}$. These changes were tabulated and their positions compared with the cusp with the largest proportional area. It appeared that commissures adjoining this cusp were particularly affected (Fig. 8).

\section{Discussion}

The pathogenesis of isolated aortic valve stenosis, in the setting of a tricuspid valve, is as yet unsolved.
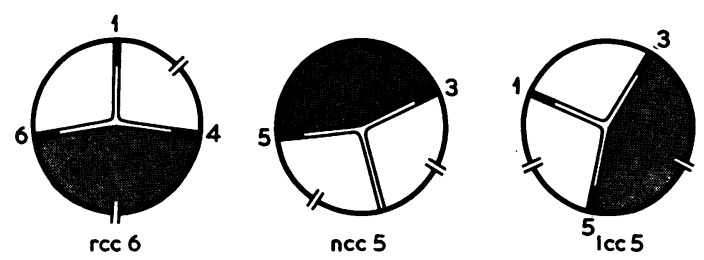

Fig. 8 Sixteen hearts with isolated aortic stenosis. The position of abnormal commissures, showing fusion of adjoining cusp leaflets over a distance of at least $5 \mathrm{~mm}$, is shown in relation to the cusp with the largest proportional area. There is preference for commissural fusion to occur at the sites adjoining the largest cusp. In the two instances where pronounced commissural fusion had occurred at a site not related to the largest cusp, it appeared that all three commissures were affected.
Various investigators have indicated, however, that the valve shows changes both at the level of the tissue matrix and at cellular level, which are compatible with an accelerated ageing process (Mönckeberg, 1904; Sohval and Gross, 1936; Hultgren 1948; McMillan and Lev, 1964; Sell and Scully, 1965; Kim and Huang, 1971; Roberts et al., 1971; Kim et al., 1976). Why should this occur in a valve that has a normal tricuspid pattern?

Roberts (1970a) suggested that minor aortic cusp inequality could underlie this condition. He examined valve cusps of unequal size in at least 3 of 49 hearts with isolated aortic stenosis. According to Roberts such differences could occasionally be observed in patients dying of non-cardiac diseases. Moreover, classical anatomical descriptions mention differences in cusp sizes, but no further consequences have been attributed to these observations. Inequalities in cusp size have also been seen in the pulmonary valve (Roberts, 1970a; Becker, 1972). However, it can be argued that unequal cusp sizes at the high-pressure left side of the heart are more significant than analogous right-sided anomalies. This intriguing concept provided the stimulus for the present study.

The results were surprising in revealing that cusps of unequal size were the rule rather than the exception. Indeed, only 5 of 200 normal hearts had cusps of equal width and height. All 195 of the remaining 'normal' hearts had cusps of different sizes, as did the 16 hearts with isolated aortic stenosis.

These results seem to contradict the earlier hypothesis. It could be argued that since cusps of unequal size are so common, their presence should be regarded as 'normal' and, therefore, could not be of significance for the pathogenesis of a relatively rare disease such as isolated tricuspid aortic stenosis. However, from an anatomical point of view one could argue that isolated aortic valve stenosis is not an uncommon finding, particularly in the elderly person, but that only the most pronounced forms are clinically overt. Indeed, practically all aortic valves in elderly people showed some degree of ageing change, though the severity of change varied considerably. Close observation, moreover, will show a striking similarity, both with regard to the gross aspect as well as to microsropy, between a large number of aortic valves of elderly patients and isolated aortic stenosis (Mönckeberg, 1904; Sohval and Gross, 1936; Hultgren, 1948; McMillan and Lev, 1964; Sell and Scully, 1965; Kim and Huang, 1971; Roberts et al., 1971; Kim et al., 1976). In isolated aortic stenosis the changes are not fundamentally different, rather they are more pronounced. One may thus consider an ageing 
process of the aortic valve leading to degrees of increasing valve abnormalities, the extreme resulting in aortic stenosis.

What role could cusps of unequal size play in this concept? It has been suggested that the ageing changes in part relate to the haemodynamic forces which act upon the valve. The congenitally bicuspid aortic valve has been suggested as an example of this and it has been thought that the increased haemodynamic stress on a bicuspid valve is responsible for the secondary changes so commonly occurring in this condition (Edwards, 1961; Roberts, 1970b). Microscopically the changes cannot be distinguished from ageing changes or from the changes that occur in isolated tricuspid aortic stenosis. If one considers the ideal situation of an equally sized tricuspid aortic valve, it is clear that the total diastolic force is equally divided over the 3 cusps. In other words, the ideally constructed valve will in itself contribute little to the development of ageing changes. However, in case of unequally sized cusps the situation is different. The total diastolic force may still be the same, but it is now no longer equally divided over the three cusps. Instead, some cusps may have to bear a greater burden than neighbouring cusps. Consequently, the process of ageing can be enhanced in one cusp as compared with the other. This may be particularly evident at the site of commissures. Most valves with isolated aortic stenosis show an eccentrically located ostium, caused by unequal involvement of cusps and commissures, and the most severely affected commissures are intimately related to the cusp with the largest proportional area (Fig. 8). The same applies to the non-stenotic, but much affected, valve in the elderly person. Such observations, in our opinion, endorse a concept that ageing changes and isolated aortic stenosis represent the same basic disorder, though to a different extent. In this concept, therefore, inequality could play an important role in enhancing the process of 'normal' ageing. Additional factors, such as haemodynamic circumstances, individual variations in tissue reactions to mechanical stress, etc. must play an important role in determining the final clinical outcome of the ageing process, but the differences in basic dimensions of the three cusps provide an anatomical substrate for a disease which, at superficial examination, seems to have affected a 'normal' tricuspid aortic valve. We, therefore, suggest that congenital variations in cusp sizes are an important factor in the pathogenesis of isolated aortic stenosis.

\section{References}

Becker, A. E. (1972). Quadricuspid pulmonary valve. Anatomical observations in 20 hearts. Acta Morphologica Neerlando-Scandinavica, 10, 299-309.

Edwards, J. E. (1961). The congenital bicuspid aortic valve. Circulation, 23, 485-488.

Hultgren, H. N. (1948). Calcific disease of the aortic valve. Archives of Pathology, 45, 694-706.

Kim, K. M., and Huang, S-N. (1971). Ultrastructural study of calcification of human aortic valve. Laboratory Investigation, 25, 357-366.

Kim, K. M., Valigrosky, J. M., Mergner, W. J., Jones, R. T., Pendergrass, R. F., and Trump, B. F. (1976). Aging changes in the human aortic valve in relation to dystrophic calcification. Human Pathology, 7, 47-60.

McMillan, J. B., and Lev, M. (1964). The aging heart. II. The valves. Fournal of Gerontology, 19, 1-14.

Mönckeberg, J. G. (1904). Der normale histologische Bau und die Sklerose der Aortenklappen. Virchows Archiv für pathologische Anatomie und Physiologie und für klinische Medizin, 176, 472-514.

Roberts, W. C. (1970a). The structure of the aortic valve in clinically isolated aortic stenosis. An autopsy study of 162 patients over 15 years of age. Circulation, 42, 91-97.

Roberts, W. C. (1970b). The congenitally bicuspid aortic valve. A study of 85 autopsy cases. American fournal of Cardiology, 26, 72-83.

Roberts, W. C., Perloff, J. K., and Costantino, T. (1971). Severe valvular aortic stenosis in patients over 65 years of age. A clinicopathologic study. American fournal of Cardio$\log y, 27,497-506$.

Sell, S., and Scully, R. E. (1965). Aging changes in the aortic and mitral valves. Histologic and histochemical studies, with observations on the pathogenesis of calcific aortic stenosis and calcification of the mitral annulus. American fournal of Pathology, 46, 345-365.

Sohval, A. R., and Gross, L. (1936). Calcific sclerosis of the aortic valve (Mönckeberg type). Archives of Pathology, 22, 477-494.

Requests for reprints to Dr. A. E. Becker, Department of Pathology, Wilhelmina Gasthuis, Eerste Helmersstraat 104, Amsterdam, The Netherlands. 